\title{
Theory of exciton dynamics in time-resolved ARPES: Intra- and intervalley scattering in two-dimensional semiconductors
}

\author{
Dominik Christiansen, ${ }^{1}$ Malte Selig, ${ }^{1}$ Ermin Malic, ${ }^{2}$ Ralph Ernstorfer $\odot,{ }^{3}$ and Andreas Knorr ${ }^{1}$ \\ ${ }^{1}$ Institut für Theoretische Physik, Nichtlineare Optik und Quantenelektronik, Technische Universität Berlin, 10623 Berlin, Germany \\ ${ }^{2}$ Department of Physics, Chalmers University of Technology, SE-412 96 Gothenburg, Sweden \\ ${ }^{3}$ Fritz-Haber-Institut der Max-Planck-Gesellschaft, Faradayweg 4-6, 14195 Berlin, Germany
}

(Received 4 July 2019; revised manuscript received 8 October 2019; published 1 November 2019)

\begin{abstract}
Time- and angle-resolved photoemission spectroscopy (trARPES) is a powerful spectroscopic method to measure the ultrafast electron dynamics directly in momentum space. However, band gap materials with exceptionally strong Coulomb interaction such as monolayer transition-metal dichalcogenides exhibit tightly bound excitons, which dominate their optical properties. This raises the question of whether excitons, in particular their formation and relaxation dynamics, can be detected in photoemissions. Here, we develop a fully microscopic theory of the temporal dynamics of excitonic time- and angle-resolved photoemission with a particular focus on the phonon-mediated thermalization of optically excited excitons to momentum-forbidden dark exciton states. We find that trARPES is able to probe the ultrafast exciton formation and relaxation throughout the Brillouin zone.
\end{abstract}

DOI: 10.1103/PhysRevB.100.205401

\section{INTRODUCTION}

We develop a theoretical description of time- and angleresolved two-photon photoemission (trARPES) signals to evaluate its potential to address the temporal dynamics of Coulomb- and phonon-induced effects on the optically excited electron and exciton dynamics. As an exemplary material, we use two-dimensional transition-metal dichalcogenides (TMDCs), which exhibit remarkable electronic and optical properties, including a direct band gap at the $K$ and $K^{\prime}$ points lying at the edges of the hexagonal Brillouin zone [1,2]. As atomically thin semiconductors, TMDCs possess a reduced dielectric screening of the Coulomb interaction, compared to the bulk case, that gives rise to the formation of a variety of excitons with binding energies of hundreds of meV [3-7]. Because of a complex electronic quasiparticle band structure [8-11], TMDCs possess a variety of optically addressable bright excitonic states as well as momentum- [12-15] and spin-forbidden $[12,13,16]$ dark excitonic states. In order to study the relaxation dynamics in this complicated excitonic landscape, different experimental techniques such as the optical pump probe [17-19], luminescence spectroscopy [20-22], and time- and angle-resolved photoemission spectroscopy [23-25] have been performed. The advantage of trARPES over pure optical experiments involving solely transitions between the valence and conduction bands is that in the latter many possible excitation and relaxation pathways contribute to the measured signal, which makes the identification of the major electronic processes difficult. Time-resolved ARPES, however, possesses a momentum resolution enabling an imaging of the Coulomb-correlated electron dynamics of an optically excited state directly in the momentum space [26-30]. In this context, for materials like monolayer TMDCs, with optical properties dominated by excitons, the question arises whether trARPES is able to discriminate between excitons as bound electron-hole pair states or electron-hole scattering (free) pair states and whether it can follow the exciton dynamics. In particular, recent theoretical studies [31-33] suggested that trARPES signals arise from the ionization of excitons: The corresponding signal is located below the conduction band minimum, reflecting the excitonic binding energy. However, so far, no description of the exciton scattering dynamics, including phonon-induced formation and thermalization observed in trARPES, is available.

In this paper, based on a many-particle Hamiltonian (Sec. II) and the Heisenberg equation of motion formalism (Sec. III), we present a fully time and angle resolved microscopic study describing the impact of Coulomb interaction between electrons and holes on the trARPES signal in two-dimensional semiconductor structures, such as TMDCs, after optical excitation. As an extension to previous studies $[33,34]$, we explicitly include not only bright, optically excitable excitons but also recently introduced momentumforbidden dark excitonic states [35] that are generated by the temporally resolved thermalization dynamics due to excitonphonon scattering and contribute to the optical line shape of TMDCs [14,36,37]. This includes $K \Lambda$ and $K K^{\prime}$ excitons with a hole at the $K$ point and an electron at the $\Lambda$ point or $K^{\prime}$ point, respectively. After optical excitation, we find first a trARPES imaging of the excitonic coherence and observe the succeeding formation of incoherent, scattering-induced, excitonic signals (Sec. IV). Our theoretical calculations reveal a method to determine the timescales of exciton formation and relaxation with direct access to momentum-forbidden dark excitonic states [35].

\section{HAMILTONIAN}

The theoretical description of the process of the twophoton photoemission (2PPE) [38-43] consists of two interfering, partly simultaneously occurring subprocesses [see 
(a)

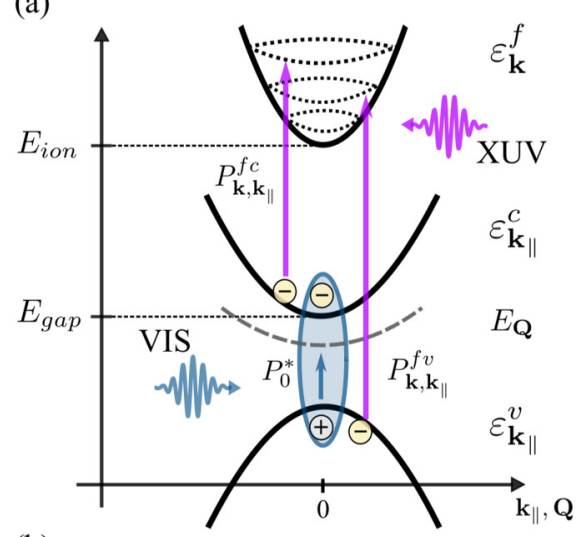

(b)

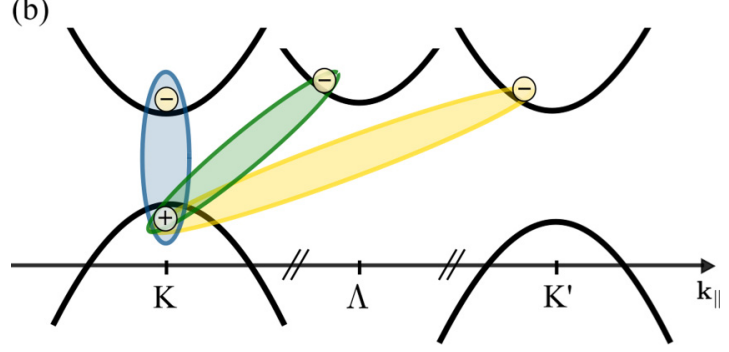

FIG. 1. (a) First, the pump pulse excites a coherent exciton, and second, the probe pulse simultaneously raises valence and conduction band electrons in the free continuum states, described by a manifold of parabolas. During the transition into the vacuum the in-plane momentum is conserved. (b) Different possible exciton states considering the complex electronic band structure, including the bright $K K$ exciton and momentum-indirect $K \Lambda$ or $K K^{\prime}$ exciton.

Fig. 1(a)]: First, the optical excitation with a visible (VIS) pump pulse close to the band edge generates correlated electron-hole pairs in the atomically thin TMDC layer (twodimensional electronic band structure for the valence and conduction bands), followed by the escape of electrons to the vacuum (three-dimensional dispersion) due to the incidence of an extreme ultraviolet (XUV) probe pulse. Because of the electron-hole interaction, the first optical pump pulse generates tightly bound electron-hole pairs-excitons (exciton momentum $\mathbf{Q}$ dispersion $E_{\mu, \mathbf{Q}}$ of state $\mu$ is shown by the dashed line). Therefore, the optical preparation and the subsequent dynamics contain valuable information about the excitonic properties and the time dynamics in the semiconductor. Figure 1(a) shows the twofold excitation scheme via the transition amplitudes $P_{\mu, \mathbf{Q}=0}^{\xi_{v} \xi_{c}}$ for the VIS (blue) and $P_{\mathbf{k}_{\|}, \mathbf{k}}^{c f \xi_{c}}$ and $P_{\mathbf{k}_{\|}, \mathbf{k}}^{v f \xi_{v}}$ for the XUV (purple) excitations. Here, only excitons with wave number $\mathbf{Q}=\mathbf{k}_{\|}^{c}-\mathbf{k}_{\|}^{v}=0$ can be optically excited by the VIS excitation. After that, excitons with $\mathbf{Q} \neq 0$, in particular incoherent excitons, can be generated only by further electronphonon scattering events (see below). It is important to note that the photon energy of the VIS pulse is smaller than the work function of the material, in contrast to the XUV pulse. Therefore, the separation of different electronic transitions excited by pump and probe pulses is ensured by their different photon energies.

To describe the trARPES signal we discuss the two contributions to the Hamiltonian: (i) the many-band TMDC part, involving the optical VIS pump pulse, and (ii) the emission process of TMDC electrons into vacuum states, involving the XUV probe pulse.

(i) The many-particle Hamiltonian for the TMDC contributions (index 1) is given by

$$
H^{(1)}=H_{0}^{(1)}+H_{\text {field }}^{(1)}+H_{c-c}^{(1)}+H_{p h}^{(1)}+H_{e l-p h}^{(1)} .
$$

The TMDC band structure contribution $H_{0}^{(1)}$,

$$
H_{0}^{(1)}=\sum_{\xi_{c}, \mathbf{k}_{\|}} \varepsilon_{\mathbf{k}_{\|}^{c \xi_{c}}}^{c} c_{\mathbf{k}_{\|}^{\dagger \xi_{c}}}^{\dagger \xi_{\mathbf{k}_{\|}}^{\xi_{c}}}+\sum_{\xi_{v}, \mathbf{k}_{\|}} \varepsilon_{\mathbf{k}_{\|} \xi_{v}}^{v \xi_{v}} v_{\mathbf{k}_{\|}}^{\dagger \xi_{v}} v_{\mathbf{k}_{\|}^{\xi_{v}}}^{\xi_{v}}
$$

contains the single-particle energies $\varepsilon_{\mathbf{k}_{\|}}^{v / c \xi_{v / c}}$ for valence $(v)$ and conduction $(c)$ band electrons treated in the effective mass approximation in the vicinity of each high-symmetry point $[9,44,45]$. The fully occupied valence band is assumed to lie at $0 \mathrm{eV}$, and the conduction band minimum is energetically situated at the free-particle band gap. Here, $c_{\mathbf{k}_{\|}^{(\dagger)} \xi_{c}}$ and $v_{\mathbf{k}_{\|}}^{(\dagger) \xi_{v}}$ annihilate (create) a conduction and valence electron in valleys $\xi_{c}$ and $\xi_{v}$ with wave number $\mathbf{k}_{\|}$, respectively. We explicitly include the high-symmetry points $K, K^{\prime}, \Lambda$, and $\Lambda^{\prime}$ [see Fig. 1(b)]. Note that the wave vector $\mathbf{k}_{\|}$is defined with respect to the corresponding valley, and the total wave vector is obtained by adding the valley wave vector $\mathbf{k}_{\|}+\boldsymbol{\xi}_{c / v}$. The spin is assumed to be equal for all electrons. In our analysis, we neglect the lower valence band at the $K$ valley as well as the $\Gamma$ valley due to the large energetic separation from the upper valence band in monolayer TMDCs [9]. The electronic excitations of the atomically thin TMDC material are confined in the $x-y$ plane $\left(\mathbf{k}_{\|}\right)$. The light-matter interaction reads

$$
H_{\text {field }}^{(1)}=\sum_{\mathbf{k}_{\|}, \xi_{c}, \xi_{v}} \mathbf{d}_{\mathbf{k}_{\|}}^{c v \xi_{c} \xi_{v}} \cdot \mathbf{E}_{v i s}(t) c_{\mathbf{k}_{\|}}^{\dagger \xi_{c}} v_{\mathbf{k}_{\|}}^{\xi_{v}} \delta_{\xi_{c}, \xi_{v}}+\text { H.c. }
$$

The optical VIS pulse $\mathbf{E}_{v i s}(t)$ generating electron-hole excitations is treated semiclassically in $\mathbf{r} \cdot \mathbf{E}$ coupling and acts as a source of the single-particle interband transition $P_{\mathbf{k}_{\|} \xi_{c}}^{\xi_{c}}=$ $\left\langle v_{\mathbf{k}_{\|}}^{\dagger \xi_{v}} c_{\mathbf{k}_{\|}}^{\xi_{c}}\right\rangle$ between the valence and conduction bands. Therefore, $\mathbf{d}_{\mathbf{k}}^{c v \xi_{c} \xi_{v}}$ describes the dipole transition matrix element, and $\mathbf{E}_{v i s}(t)$ denotes the optical pump pulse. For the optically induced interband transition only wave number vertical transitions occur $\left(\mathbf{k}_{\|}^{v}=\mathbf{k}_{\|}^{c}\right.$ and $\left.\xi_{v}=\xi_{c}\right)$, and the sum over the valley indices is restricted to the $K$ and $K^{\prime}$ points, considering an optical near-gap excitation of the TMDC monolayer. The Coulomb interaction between electrons and holes is included by

$$
H_{c-c}^{(1)}=\frac{1}{2} \sum_{\substack{\xi_{c}, \xi_{v}, \mathbf{k}_{\|}, \mathbf{k}_{\|}^{\prime}, \mathbf{q}}} V_{\mathbf{k}_{\|}, \mathbf{k}_{\|}^{\prime}, \mathbf{q}}^{v c \xi_{\xi^{\prime}} \xi_{c}} c_{\mathbf{k}_{\|}+\mathbf{q}}^{\dagger \xi_{c}} v_{\mathbf{k}_{\|}^{\prime}-\mathbf{q}}^{\dagger \xi_{v}} v_{\mathbf{k}_{\|}^{\prime}}^{\xi_{v}} c_{\mathbf{k}_{\|}}^{\xi_{c}}+\text { H.c. }
$$

with the matrix element $V_{\mathbf{k}_{\|}, \mathbf{k}_{\|}^{\prime}, \mathbf{q}}^{v c \xi_{v} c_{c}}=V_{\mathbf{q}} \int d^{3} r \int d^{3} r^{\prime} \psi_{\mathbf{k}_{\|}^{\prime}}^{* v \xi_{v}}(\mathbf{r})$ $\psi_{\mathbf{k}_{\|}}^{* c \xi_{c}}\left(\mathbf{r}^{\prime}\right) e^{i \mathbf{q}\left(\mathbf{r}-\mathbf{r}^{\prime}\right)} \psi_{\mathbf{k}_{\|}^{\prime}}^{v \xi_{v}}(\mathbf{r}) \psi_{\mathbf{k}_{\|}}^{c \xi_{c}}\left(\mathbf{r}^{\prime}\right)[46,47]$. Here, the Coulomb potential is treated by an analytical model of the dielectric function within a dielectric environment, including the nonlinear $\mathbf{q}$ dependency, beyond the Rytova-Keldysh approximation [48-51]. Since TMDC excitons are spectrally stable over a wide doping and excitation range [52,53], a static treatment of the Coulomb potential captures well all excitonic effects in the low-density regime. This is different from metals, where 
excitonic excitations are built up on top of an interacting electron gas [54,55]. The details of the introduced electronic wave functions $\psi_{\mathbf{k}_{\|} / c \xi_{v / c}}^{v}$, the transition dipole element, and the Coulomb matrix element are discussed in the Supplemental Material [56]. To include dissipation, i.e., incoherent exciton dynamics such as exciton density formation and relaxation [35], we include the electron-phonon interaction in the TMDC layer:

$$
\begin{aligned}
H_{p h}^{(1)}+ & H_{e l-p h}^{(1)} \\
= & \sum_{\xi, \alpha, \mathbf{K}} \hbar \Omega_{\mathbf{K}}^{\xi \alpha} b_{\mathbf{K}}^{\dagger \alpha \xi} b_{\mathbf{K}}^{\alpha \xi} \\
& +\sum_{\substack{\xi_{c}, \xi_{c}^{\prime}, \alpha, \mathbf{k}_{\|}, \mathbf{K}}}\left(g_{\mathbf{K}}^{c \xi_{c} \xi_{c}^{\prime} \alpha} c_{\mathbf{k}_{\|}+\xi_{c}}^{\dagger \xi_{c}} c_{\mathbf{k}_{\|}}^{\xi_{c}^{\prime}}\right)\left(b_{\mathbf{K}}^{\alpha \xi_{c}-\xi_{c}^{\prime}}+b_{-\mathbf{K}}^{\dagger \alpha \xi_{c}^{\prime}-\xi_{c}}\right) \\
& +\sum_{\substack{\xi_{v}, \xi_{v}^{\prime}, \alpha, \mathbf{k}_{\|}, \mathbf{K}}}\left(g_{\mathbf{K}}^{v \xi_{v} \xi_{v}^{\prime} \alpha} v_{\mathbf{k}_{\|}+\mathbf{K}}^{\dagger \xi_{v}} v_{\mathbf{k}_{\|} \|}^{\xi_{v}^{\prime}}\right)\left(b_{\mathbf{K}}^{\alpha \xi_{v}-\xi_{v}^{\prime}}+b_{-\mathbf{K}}^{\dagger \alpha \xi_{v}^{\prime}-\xi_{v}}\right) .
\end{aligned}
$$

Here, $b_{\mathbf{K}}^{(\dagger) \alpha \xi}$ denotes the annihilation (creation) of a phonon with mode $\alpha$ at the valley $\xi$ and two-dimensional wave vector $\mathbf{K} . g_{\mathbf{K}}^{c / v \xi_{c / v} \xi_{c / v}^{\prime} \alpha}$ is the electron-phonon matrix element for electronic transitions from the valley $\xi_{c / v}^{\prime}$ to the valley $\xi_{c / v}$ in the conduction or valence band. The Hamiltonian describes electrons scattering from $\xi_{c / v}^{\prime}+\mathbf{k}_{\|}$to $\xi_{c / v}+\mathbf{k}_{\|}+\mathbf{K}$ under absorption (emission) of phonons with wave vector $\xi_{c / v}-\xi_{c / v}^{\prime}+$ $\mathbf{K}\left(-\xi_{c / v}+\xi_{c / v}^{\prime}-\mathbf{K}\right)$. The electron-phonon interaction takes into account intra- and intervalley scattering of electrons with an effective deformation potential approximation. We include two acoustic (LA, TA) and two optical (LO, TO) phonon modes, which show the strongest deformation coupling to electrons [57-60].

(ii) The next contribution to the Hamiltonian describes the emission process of Coulomb-correlated electrons into the vacuum initiated by a XUV pulse. In the following, $\varepsilon_{\mathbf{k}}^{f}$ denotes the dispersion of the free-electron continuum above the ionization threshold $E_{\text {ion }}$ of the TMDC [61,62]. The transitions from the semiconductor to the vacuum states are induced by a XUV probe pulse $\mathbf{E}_{\mathrm{xuv}}(t)$. We introduce the electron operators $f_{\mathbf{k}}^{(\dagger)}$ annihilating (creating) an electron in the three-dimensional continuum states of the vacuum:

$$
\begin{gathered}
H_{0}^{(2)}=\sum_{\mathbf{k}} \varepsilon_{\mathbf{k}}^{f} f_{\mathbf{k}}^{\dagger} f_{\mathbf{k}}, \\
H_{\text {field }}^{(2)}=\sum_{\xi_{c}, \mathbf{k}^{\prime}} \mathbf{d}_{\mathbf{k}^{\prime}}^{c f \xi_{c}} \delta_{\mathbf{k}_{\|}, \mathbf{k}_{\|}^{\prime}} \cdot \mathbf{E}_{\mathrm{xuv}}(t) c_{\mathbf{k}_{\|}^{\dagger \xi_{c}}} f_{\mathbf{k}^{\prime}} \\
+\sum_{\xi_{v}, \mathbf{k}^{\prime}} \mathbf{d}_{\mathbf{k}^{\prime}}^{v f \xi_{v}} \delta_{\mathbf{k}_{\|}, \mathbf{k}_{\|}^{\prime}} \cdot \mathbf{E}_{\mathrm{xuv}}(t) v_{\mathbf{k}_{\|}}^{\dagger \xi_{v}} f_{\mathbf{k}^{\prime}}+\text { H.c. }
\end{gathered}
$$

The continuum of the vacuum states is described by a threedimensional wave vector $\mathbf{k} \in \mathbb{R}^{3}$. In the course of electron spectroscopy, the probe pulse excites electrons from the valence and conduction bands to the vacuum states via the transition amplitudes $P_{\mathbf{k}, \mathbf{k}_{\|}^{v f \xi_{v}}}^{v \xi_{\|}}=\left\langle v_{\mathbf{k}_{\|}}^{\dagger \xi_{v}} f_{\mathbf{k}}\right\rangle$ and $P_{\mathbf{k}, \mathbf{k}_{\|}}^{c f \xi_{c}}=\left\langle c_{\mathbf{k}_{\|}}^{\dagger \xi_{c}} f_{\mathbf{k}}\right\rangle$, respectively. Here, the dipole matrix reads $\mathbf{d}_{\mathbf{k}}^{c / v f \xi_{c / v}}=$ $-i e \int d^{3} r \psi_{\mathbf{k}_{\|}}^{* c / v \xi_{c / v}} \nabla_{\mathbf{k}} \psi_{\mathbf{k}}^{f}$, where $\mathbf{k}=\left(\mathbf{k}_{\|}, k_{z}\right)$. The conservation of the in-plane momentum $\delta_{\mathbf{k}_{\|}, \mathbf{k}_{\|}^{\prime}}$ follows directly from the optical matrix element [63]. From the emitted photoelectron distribution $\rho_{\mathbf{k}}^{f}=\left\langle f_{\mathbf{k}}^{\dagger} f_{\mathbf{k}}\right\rangle$, due to the conservation of the inplane wave vector, we obtain information about the wave number distribution of valence and conduction band electrons inside the TMDC material.

\section{DYNAMICAL EQUATIONS}

In trARPES experiments the detector measures the photocurrent of electrons, which are emitted by the probe pulse in a certain direction sensitive to their kinetic energy. Using the Heisenberg equation of motion, we develop a description of the time- and angle-resolved photoemitted vacuum electron distribution [64-67] as a function of energy $\varepsilon_{\mathbf{k}}^{f}$ and in-plane wave number $\mathbf{k}_{\|}$:

$$
I_{\mathbf{k}_{\|}, \varepsilon_{\left(\mathbf{k}_{\|}, k_{z}\right)}^{f}}(\tau)=\lim _{t \rightarrow \infty} \int_{-\infty}^{t} d t^{\prime} \partial_{t^{\prime}} \rho_{\mathbf{k}}^{f}\left(t^{\prime}, \tau\right),
$$

where $\rho_{\mathbf{k}}^{f}(t, \tau)$ is the vacuum electron density depending on real time $t$ and the time delay $\tau$ between the VIS and XUV pulse. Since the time integral extends to $\infty$, we count all photoelectrons, which reach the detector. In principle, to derive the two-photon photoemission signal, a detector model must be considered. Here, defining Eq. (8) as the signal, we assume a detector sensitive to the final-state occupation to be the 2PPE signal [43]. We note that the time-integrated finalstate occupation has been used as an alternative definition of the photoemission signal assuming a detector sensitive to the total electron number [68,69]. We expect, however, at least for our calculations, equally informative results for both limiting situations. The occurring situation is similar to problems in the definition of nonstationary signals via photon detection [70] with simultaneous energy and time resolution.

In the following, for all equations the rapid carrier frequency pulse oscillation contribution has been split off in a rotating wave approximation for each pulse. For the vacuum electron distribution, which determines the observable in Eq. (8), we find

$$
\frac{d}{d t} \rho_{\mathbf{k}}^{f}=-2 \operatorname{Im}\left(\Omega_{\mathbf{k}}^{v f \xi_{v}} P_{\mathbf{k}_{\|}, \mathbf{k}}^{v f \xi_{v}}+\Omega_{\mathbf{k}}^{c f \xi_{c}} P_{\mathbf{k}_{\|}, \mathbf{k}}^{c f \xi_{c}}\right),
$$

where we define the Rabi frequency $\Omega_{\mathbf{k}}^{c / v f \xi_{c / v}}(t)=d_{\mathbf{k}}^{c / v f \xi_{c / v}}$ $E_{\mathrm{xuv}}(t) / \hbar$ and $E_{\mathrm{xuv}}(t)$ as the XUV pulse envelope. Equation (9) shows that the source of the vacuum electrons is the transition amplitudes $P_{\mathbf{k}, \mathbf{k}_{\|}}^{c f \xi_{c}}$ and $P_{\mathbf{k}, \mathbf{k}_{\|}}^{v f \xi_{v}}$ between conduction and valence bands to the vacuum, referred to as the photoemission process [see Fig. 1(a)].

In order to take into account the electron-hole Coulomb coupling for the conduction band electrons in Eq. (9) we insert a unit operator $\mathbb{1}=|0\rangle\langle 0|+\sum_{\xi_{v}, \mathbf{k}_{\|}^{v}} \sum_{\mathbf{k}_{\|}^{v}}^{\xi_{v}^{v}} v_{\mathbf{k}_{\|}^{v}}^{\dagger \xi_{v}}+$ $\sum_{\xi_{c}, \mathbf{k}_{\|}^{c}} c_{\mathbf{k}_{\|}^{c}}^{\dagger \xi_{c}} \sum_{\mathbf{k}_{\|}^{c}}^{\xi_{c}}+\mathcal{O}\left(n a_{B}^{2}\right)$ exploiting the completeness relation of the Fock space [71-74]. $n$ denotes the pair (surface) density, and $a_{B}$ is the exciton Bohr radius. Higher-order contributions to the unit operator are neglected since we restrict ourselves to the low-density regime. $|0\rangle$ denotes the ground states of the semiconductor with completely filled valence and empty 
conduction bands. The expansion of $c_{\mathbf{k}_{\|} \xi_{c}} f_{\mathbf{k}}$ in Eq. (9), by inserting the unit operator, yields

$$
c_{\mathbf{k}_{\|}}^{\dagger \xi_{c}} f_{\mathbf{k}}=\sum_{\xi_{v}, \mathbf{k}_{\|}^{v}} c_{\mathbf{k}_{\|}}^{\dagger \xi_{c}} v_{\mathbf{k}_{\|}^{v_{v}}}^{\dagger v_{\mathbf{k}_{\|}^{v}}^{\dagger \xi_{v}}} f_{\mathbf{k}} \text {. }
$$

Since we consider the optical excitation of undoped semiconductors below the free-particle band gap, only the second contribution to the unit operator is relevant. With this procedure the conduction electron operators are expressed uniquely by electron-hole pair operators. To treat the quantum-mechanical hierarchy problem arising from the many-particle interaction we exploit the cluster expansion scheme [75-77]. Analogously, the valence band electrons in $v_{\mathbf{k}_{\|}}^{\dagger \xi_{v}} f_{\mathbf{k}}$ of Eq. (9) need

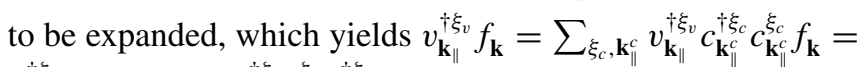
$v_{\mathbf{k}_{\|}}^{\dagger \xi_{v}} f_{\mathbf{k}}-\sum_{\xi_{c}, \mathbf{k}_{\|}^{c}} v_{\mathbf{k}_{\|}^{\dagger \xi_{0}}}^{\dagger \xi_{\mathbf{k}_{\|}^{c}}^{\xi_{c}}} \overbrace{\mathbf{k}_{\|}^{c}}^{\dagger \xi_{c}} f_{\mathbf{k}}$. Here, the interband Coulomb interaction leads to corrections to the dominating $v_{\mathbf{k}_{\|}}^{\dagger \xi_{v}} f_{\mathbf{k}}$ term. Since we restrict our analysis to the leading order justified by a weak optical excitation of the sample such that the valence band occupation $\rho_{\mathbf{k}_{\|}}^{v \xi_{v}} \approx 1$ holds for all investigated scenarios, we can neglect the Coulomb-induced contribution here. For the vacuum electron density $\rho_{\mathbf{k}}^{f}$ including Coulomb coupling we find

$$
\begin{aligned}
\frac{d}{d t} \rho_{\mathbf{k}}^{f}= & -2 \operatorname{Im}\left(\Omega_{\mathbf{k}}^{v f \xi_{v}} P_{\mathbf{k}_{\|}, \mathbf{k}}^{v f \xi_{v}}+\Omega_{\mathbf{k}}^{c f \xi_{c}} P_{\mathbf{k}_{\|} \xi_{c} \xi_{v}} P_{\mathbf{k}_{\|}, \mathbf{k}}^{v f \xi_{v}} e^{-\frac{1}{i \hbar} \varepsilon_{v i s} t}\right. \\
& \left.+\sum_{\xi_{v}, \mathbf{k}_{\|}^{v}} \Omega_{\mathbf{k}}^{c f \xi_{c}} \delta\left\langle P_{\mathbf{k}, \mathbf{k}_{\|}^{\dagger \xi_{v}}}^{\dagger \xi_{v}} P_{\mathbf{k}_{\|}^{v}, \mathbf{k}}^{v f \xi_{v}}\right\rangle\right) .
\end{aligned}
$$

The first term accounts for the photoemission of valence band electrons. The second term stems from interband Coulomb interaction in Hartree-Fock limit and couples the interband transition with the transition between the valence band and vacuum. The third term is a correlated two-particle quantity $\delta\left\langle c_{\mathbf{k}_{\|}+\mathbf{q}}^{\dagger \xi_{c}} v_{\mathbf{k}_{\|}^{\nu}+\mathbf{q}}^{\xi_{v}} v_{\mathbf{k}_{\|}^{v}}^{\dagger \xi_{v}} f_{\mathbf{k}}\right\rangle$, describing the Coulomb-correlated photoemission, obtained beyond the Hartree-Fock limit, $\delta\left\langle a_{1}^{\dagger} a_{2}^{\dagger} a_{3} a_{4}\right\rangle=\left\langle a_{1}^{\dagger} a_{3}\right\rangle\left\langle a_{2}^{\dagger} a_{4}\right\rangle-$ $\left\langle a_{1}^{\dagger} a_{4}\right\rangle\left\langle a_{2}^{\dagger} a_{3}\right\rangle+\left\langle a_{1}^{\dagger} a_{2}^{\dagger} a_{3} a_{4}\right\rangle$. While in the lowest Hartree-Fock level all correlations between the carriers are neglected, in the first order the appearing correlated quantity contains the true two-body interaction describing deviations from the factorization. This term includes a sum over the valence band electrons [see Eq. (11)]. The kinetics of the photoemission of valence band electrons reads

$$
\frac{d}{d t} P_{\mathbf{k}_{\|}, \mathbf{k}}^{v f \xi_{v}}=\frac{1}{i \hbar}\left(\varepsilon_{\mathbf{k}}^{f}-\varepsilon_{\mathbf{k}_{\|} \xi_{v}}^{v \xi_{x}}-\varepsilon_{\mathrm{xuv}}\right) P_{\mathbf{k}_{\|}, \mathbf{k}}^{v f \xi_{v}}+\left.\partial_{t} P_{\mathbf{k}_{\|}, \mathbf{k}}^{v f \xi_{c}}\right|_{\text {scatt }}-i \Omega_{\mathbf{k}}^{f v \xi_{v}} \rho_{\mathbf{k}_{\|}}^{v \xi_{v}}-i \Omega_{\mathbf{k}}^{f c \xi_{c}} P_{\mathbf{k}_{\|} \xi_{\|} \xi_{c}} e^{\frac{1}{i \hbar} \varepsilon_{v i s} t}
$$

The solution (12) oscillates with the kinetic energy of the vacuum and valence band electrons and is driven by the electronic valence band occupation. Schematically, using the notation $\left.\partial_{t} P_{\mathbf{k}_{\|}, \mathbf{k}}^{v f \xi_{c}}\right|_{s c a t t}$, we include phonons, which lead to a dephasing and a broadening $\gamma$ of the transition.

The equation of motion of the correlated two-particle quantity reads

$$
\begin{aligned}
& \frac{d}{d t} \delta\left\langle P_{\mathbf{k}_{\|}, \mathbf{k}_{\|}^{v}}^{\dagger \xi_{\xi} \xi_{v}} P_{\mathbf{k}_{\|}^{v}, \mathbf{k}}^{v f \xi_{v}}\right\rangle=\frac{1}{i \hbar}\left(\varepsilon_{\mathbf{k}}^{f}+\varepsilon_{\mathbf{k}_{\|}^{v}}^{v \xi_{v}}-\varepsilon_{\mathbf{k}_{\|}^{v}}^{v \xi_{v}}-\varepsilon_{\mathbf{k}_{\|}}^{c \xi_{c}}-\varepsilon_{\mathrm{xuv}}\right) \delta\left\langle P_{\mathbf{k}_{\|}, \mathbf{k}_{\|}^{v}}^{\dagger \xi_{\xi} \xi_{v}} P_{\mathbf{k}_{\|}^{v}, \mathbf{k}}^{v f \xi_{v}}\right\rangle+\partial_{t} \delta\left\langle\left. P_{\mathbf{k}_{\|}, \mathbf{k}_{\|}^{v}}^{\dagger \xi_{\xi} \xi_{v}} P_{\mathbf{k}_{\|}^{v}, \mathbf{k}}^{v f \xi_{v}}\right|_{\text {scatt }}\right.
\end{aligned}
$$

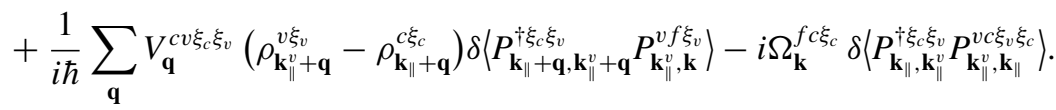

The source terms of Eq. (13) are TMDC-interband Coulomb correlations $\delta\left\langle P_{\mathbf{k}_{\|}, \mathbf{k}_{\|}^{v}}^{\dagger \xi_{c} \xi_{v}} P_{\mathbf{k}_{\|}^{v}, \mathbf{k}_{\|}}^{v c \xi_{v} \xi_{c}}\right\rangle$. The vacuum electron-induced Pauli blocking of the transition (13) is assumed to be small compared to the electron-hole population and is therefore neglected. For the TMDC interband transitions also occurring in Eq. (11) we obtain

$$
\begin{aligned}
\frac{d}{d t} P_{\mathbf{k}_{\|} \xi_{\|} \xi_{c}}= & \frac{1}{i \hbar}\left(\varepsilon_{\mathbf{k}_{\|}^{c \xi_{c}}}^{\xi_{\|}}-\varepsilon_{\mathbf{k}_{\|}}^{v \xi_{v}}-\varepsilon_{v i s}\right) P_{\mathbf{k}_{\|} \xi_{v} \xi_{c}}^{\xi_{0}} \\
& +\left.\partial_{t} P_{\mathbf{k}_{\|}}^{\xi_{v} \xi_{c}}\right|_{\text {scatt }}-i \Omega_{\mathbf{k}_{\|}}^{c v \xi_{c} \xi_{v}}\left(\rho_{\mathbf{k}_{\|}}^{v \xi_{v}}-\rho_{\mathbf{k}_{\|}}^{c \xi_{c}}\right) \delta_{\xi_{v}, \xi_{c}} \\
& -\frac{1}{i \hbar} \sum_{\mathbf{q}} V_{\mathbf{q}}^{v c \xi_{v} \xi_{c}}\left(\rho_{\mathbf{k}_{\|}}^{v \xi_{v}}-\rho_{\mathbf{k}_{\|}}^{c \xi_{c}}\right) P_{\mathbf{k}_{\|} \xi_{v} \xi_{c}} . \mathbf{q}
\end{aligned}
$$

The attractive Coulomb interaction $V_{\mathbf{q}}^{v c \xi_{v} \xi_{c}}$ leads to a renormalization of the Rabi frequency of the exciting field [78] and is treated by the Wannier equation after a coordinate transformation into the exciton basis. Since only the edges of the hexagonal Brillouin zone are optically excited and only momentum vertical transitions are valid, the electron and hole valleys of the TMDC interband polarization are restricted to $K$ and $K^{\prime}$, respectively.

To transfer the equations to the excitonic basis [46,74], we introduce the center of mass momentum $\mathbf{Q}=\mathbf{k}_{\|}^{c}-\mathbf{k}_{\|}^{v}$ and the relative momentum $\mathbf{q}_{\|}=\alpha_{\xi_{v}}^{\xi_{c}} \mathbf{k}_{\|}^{v}+\beta_{\xi_{v}}^{\xi_{c}} \mathbf{k}_{\|}^{c}$ with the mass factors $\alpha_{\xi_{v}}^{\xi_{c}}=m_{h}^{\xi_{v}} /\left(m_{e}^{\xi_{c}}+m_{h}^{\xi_{v}}\right)$ and $\beta_{\xi_{v}}^{\xi_{c}}=m_{e}^{\xi_{c}} /\left(m_{h}^{\xi_{v}}+m_{e}^{\xi_{c}}\right)$. For equal electron and hole momenta the relative momentum equals the electron momentum. In this basis, we exploit the Wannier equation $[46,78,79]$,

$$
\frac{\hbar^{2} \mathbf{q}_{\|}^{2}}{2 m} \varphi_{\mu, \mathbf{q}_{\|} \xi_{c}}-\sum_{\mathbf{k}_{\|}} V_{\mathbf{k}_{\|}}^{v c \xi_{v} \xi_{c}} \varphi_{\mu, \mathbf{q}_{\|}+\mathbf{k}_{\|}}^{\xi_{v} \xi_{c}}=E_{B}^{\mu \xi_{v} \xi_{c}} \varphi_{\mu, \mathbf{q}_{\|}}^{\xi_{v} \xi_{c}},
$$

to access the exciton wave function $\varphi_{\mu, \mathbf{q}_{\|}}^{\xi_{v} \xi_{c}}$ and binding energy $E_{B}^{\mu \xi_{v} \xi_{c}}$ of the exciton state $\mu$. The reduced mass of the exciton is denoted by $m$. By expanding the excitonic polarization into solutions of the Wannier equation $P_{\mathbf{q}_{\|}, \mathbf{Q}}^{\xi_{v} \xi_{c}}=\sum_{\mu} \varphi_{\mu, \mathbf{q}_{\|}}^{\xi_{v} \xi_{c}} P_{\mu, \mathbf{Q}}^{\xi_{v} \xi_{c}}$ the relative coordinate $\mathbf{q}_{\|}$can be eliminated, and the exciton dynamics is described by the center of mass momentum $\mathbf{Q}$. 
Using this expansion, we provide Eqs. (11)-(14) in the exciton basis.

In the limit of negligible Pauli blocking, we obtain for the excitonic transition (14)

$$
\begin{aligned}
\frac{d}{d t} P_{\mu, \mathbf{Q}}^{\xi_{u} \xi_{c}}= & \frac{1}{i \hbar}\left(E_{\mu, \mathbf{Q}}^{\xi_{v} \xi_{c}}-\varepsilon_{v i s}\right) P_{\mu, \mathbf{Q}}^{\xi_{u} \xi_{c}}+\left.\partial_{t} P_{\mu, \mathbf{Q}}^{\xi_{u} \xi_{c}}\right|_{\text {scatt }} \\
& -i \Omega_{\mu}^{\xi_{v} \xi_{c}}(t) \delta_{\mathbf{Q}, 0}^{\xi_{v}, \xi_{c}},
\end{aligned}
$$

with the excitonic Rabi frequency $\Omega_{\mu}^{\xi_{v} \xi_{c}}(t)$ and the exciton kinetic energy, which reads $E_{\mu, \mathbf{Q}}^{\xi_{v} \xi_{c}}=\hbar^{2} \mathbf{Q}^{2} / 2 M^{\xi_{v} \xi_{c}}+$ $E_{\mu}^{\xi_{v} \xi_{c}}$, where $M^{\xi_{v} \xi_{c}}=m_{h}^{\xi_{v}}+m_{e}^{\xi_{c}}$ denotes the exciton mass and $E_{\mu}^{\xi_{u} \xi_{c}}=E_{\text {gap }}^{\xi_{v} \xi_{c}}+E_{B}^{\mu \xi_{v} \xi_{c}}$ is the exciton energy. Because of the assumed perpendicular excitation geometry only excitons with a vanishing center of mass momentum couple to the light field. The phonon contribution leads to a dephasing of the excitonic transition and to the formation of incoherent excitons $N_{\mu, \mathbf{Q}}^{\xi_{v} \xi_{c}}=\delta\left\langle P_{\mu, \mathbf{Q}}^{\dagger \xi_{c} \xi_{v}} P_{\mu, \mathbf{Q}}^{\xi_{v} \xi_{c}}\right\rangle$. A detailed derivation of the exciton-phonon interaction and the microscopic computation of the dephasing can be found in Refs. [14,35,36]. The used electron-phonon parameters stem from first-principles calculations described in Refs. $[57,59,60]$. The phonon reservoir is treated in the bath approximation following a Bose distribution.

For the photoemission signal (11), now in the exciton basis, we obtain

$$
\begin{aligned}
\frac{d}{d t} \rho_{\mathbf{k}}^{f}= & -2 \operatorname{Im}\left(\Omega_{\mathbf{k}}^{v f \xi_{v}} P_{\mathbf{k}_{\|}, \mathbf{k}}^{v f \xi_{v}}+\sum_{\mu} \Omega_{\mu, \mathbf{k}}^{c f \xi_{c}-} P_{\mu, 0}^{* \xi_{c} \xi_{v}} P_{\mathbf{k}_{\|, \mathbf{k}}}^{v f \xi_{v}}\right. \\
& \left.+\sum_{\mu, \xi_{v}, \mathbf{Q}} \Omega_{\mu, \mathbf{k}, \mathbf{Q}}^{c f \xi_{c}} \delta\left\langle P_{\mu, \mathbf{Q}}^{\dagger \xi_{c} \xi_{v}} P_{\mathbf{k}_{\|}-\mathbf{Q}, \mathbf{k}}^{v f \xi_{v}}\right)\right)
\end{aligned}
$$

with the coupling elements $\Omega_{\mu, \mathbf{k}}^{c f \xi_{c}-}=\Omega_{\mathbf{k}}^{c f \xi_{c}} \varphi_{\mu, \mathbf{k}_{\|}}^{* \xi_{c} \xi_{v}} e^{-\frac{1}{i \hbar} \varepsilon_{v i s} t}$ and $\Omega_{\mu, \mathbf{k}, \mathbf{Q}}^{c f \xi_{c}}=\Omega_{\mathbf{k}}^{c f \xi_{c}} \varphi_{\mu, \mathbf{k}_{\|}-\alpha_{\xi v}^{\xi_{c}}}^{* \xi_{c} \xi_{v}}$. Now, we see that the photoemission of conduction band electrons always occurs via excitonic states. The TMDC interband Coulomb interaction couples, through the Fock term, the coherently driven excitonic transition $P_{\mu, 0}^{* \xi_{\xi} \xi_{v}}$ with the transition between the valence band and vacuum (the coherent source is present only for coherent excitons and XUV field), and most importantly, as a source of Coulomb correlations, beyond the Hartree-Fock approximation we identify the exciton-assisted photoemission transition $\delta\left\langle P_{\mu, \mathbf{Q}}^{\dagger \xi_{c} \xi_{v}} P_{\mathbf{k}_{\|}-\mathbf{Q}, \mathbf{k}}^{v f \xi_{v}}\right\rangle:$

$$
\begin{aligned}
\frac{d}{d t} \delta\left\langle P_{\mu, \mathbf{Q}}^{\dagger \xi_{c} \xi_{v}} P_{\mathbf{k}_{\|}-\mathbf{Q}, \mathbf{k}}^{v f \xi_{v}}\right\rangle= & \frac{1}{i \hbar} \Delta \varepsilon_{\mathbf{k}_{\|}, \mathbf{Q}, k_{z}} \delta\left\langle P_{\mu, \mathbf{Q}}^{\dagger \xi_{c} \xi_{v}} P_{\mathbf{k}_{\|}-\mathbf{Q}, \mathbf{k}}^{v f \xi_{v}}\right\rangle \\
& +\left.\partial_{t} \delta\left\langle P_{\mu, \mathbf{Q}}^{\dagger \xi_{c} \xi_{v}} P_{\mathbf{k}_{\|}-\mathbf{Q}, \mathbf{k}}^{v f \xi_{v}}\right\rangle\right|_{\text {scatt }} \\
& -i \sum_{\lambda} \tilde{\Omega}_{\mu, \lambda, \mathbf{k}_{\|}, \mathbf{Q}}^{f c \xi_{v} \xi_{c}} N_{\lambda, \mathbf{Q}}^{\xi_{v} \xi_{c}},
\end{aligned}
$$

with $\Delta \varepsilon_{\mathbf{k}_{\|}, \mathbf{Q}, k_{z}}=\varepsilon_{\mathbf{k}}^{f}-\varepsilon_{\mathbf{k}_{\|}-\mathbf{Q}}^{v \xi_{v}}-E_{\mu, \mathbf{Q}}^{\xi_{v} \xi_{c}}-\varepsilon_{\text {xuv }}$ and $\tilde{\Omega}_{\mu, \lambda, \mathbf{k}, \mathbf{Q}}^{f c \xi_{\nu} \xi_{c}}=$ $\Omega_{\mathbf{k}}^{f c \xi_{c}}\left|\varphi_{\lambda, \mathbf{k}_{\|}-\alpha_{\xi_{v}}^{\xi_{c}} \mathbf{Q}}^{\xi_{1} \xi_{c}}\right|^{2} \varphi_{\mu, \mathbf{k}_{\|}-\alpha_{\xi_{v}}^{\xi_{c}}}^{\xi_{v} \xi_{c}}$. The oscillation energy of the TMDC exciton-assisted valence-band-vacuum transition carries the exciton energy, is driven by the incoherent exciton density, and therefore carries the information about the bound
TABLE I. Band structure parameters for $\mathrm{WSe}_{2}$ obtained from first-principles calculations $[9,61]$ and the resulting excitonic parameters (restricted to spin up).

\begin{tabular}{llcl}
\hline \hline Parameter & Value & Parameter & Value \\
\hline$m_{e}^{K} / m_{0}$ & 0.29 & $M_{K K} / m_{0}$ & 0.65 \\
$m_{e}^{\Lambda} / m_{0}$ & 0.56 & $M_{K \Lambda} / m_{0}$ & 0.92 \\
$m_{h}^{K} / m_{0}$ & 0.36 & $M_{K K^{\prime}} / m_{0}$ & 0.76 \\
$E_{\mathrm{gap}}^{K K} / \mathrm{eV}$ & 2.08 & $E_{B 1}^{K K} / \mathrm{eV}$ & 0.23 \\
$E_{\mathrm{gap}}^{K \Lambda} / \mathrm{eV}$ & 2.075 & $E_{B 1 s}^{K \Lambda} / \mathrm{eV}$ & 0.26 \\
$E_{\mathrm{gap}}^{K K^{\prime}} / \mathrm{eV}$ & 2.057 & $E_{B 1 s}^{K K^{\prime}} / \mathrm{eV}$ & 0.25 \\
$E_{\mathrm{gap}}^{K \Lambda^{\prime}} / \mathrm{eV}$ & 2.208 & $E_{B 1 s}^{K \Lambda^{\prime}} / \mathrm{eV}$ & 0.28 \\
$E_{\text {ion }} / \mathrm{eV}$ & 5.17 & $\epsilon_{S i O_{2}}$ & 3.9 \\
\hline \hline
\end{tabular}

TMDC excitons and their incoherent scattering dynamics. For completeness we also provide the equation of motion for $P_{\mathbf{k}_{\|}, \mathbf{k}}^{v f \xi_{v}}$ in Eq. (17):

$$
\begin{aligned}
\frac{d}{d t} P_{\mathbf{k}_{\|}, \mathbf{k}}^{v f \xi_{v}}= & \frac{1}{i \hbar}\left(\varepsilon_{\mathbf{k}}^{f}-\varepsilon_{\mathbf{k}_{\|}}^{v \xi_{v}}-\varepsilon_{\text {xuv }}\right) P_{\mathbf{k}_{\|}, \mathbf{k}}^{v f \xi_{v}}+\left.\partial_{t} P_{\mathbf{k}_{\|}, \mathbf{k}}^{v f \xi_{v}}\right|_{\text {scatt }} \\
& -i \Omega_{\mathbf{k}}^{f v \xi_{v}} \rho_{\mathbf{k}_{\|}}^{v \xi_{v}}-i \sum_{\mu} \Omega_{\mu, \mathbf{k}}^{f c \xi_{c}+} P_{\mu, 0}^{\xi_{v} \xi_{c}},
\end{aligned}
$$

with $\Omega_{\mu, \mathbf{k}}^{f c \xi_{c}+}=\Omega_{\mathbf{k}}^{f c \xi_{c}} \varphi_{\mu, \mathbf{k}_{\|}}^{\xi_{v} \xi_{c}} e^{\frac{1}{i \hbar} \varepsilon_{v i s} t}$

Next, we investigate the photoemission signal (8) by analyzing its individual contributions. As a first attack, we focus on the lowest-lying $1 s A$ state $P_{\mu, 0}^{\xi_{v} \xi_{c}} \rightarrow P_{0}^{\xi_{v} \xi_{c}}$ and $N_{\lambda, \mathbf{Q}}^{\xi_{v} \xi_{c}} \rightarrow$ $N_{\mathbf{Q}}^{\xi_{v} \xi_{c}}$, justified by the large $1 s-2 s$ separation in comparison to the thermal energy introduced by exciton-phonon scatteringmediated thermalization $[80,81]$

\section{TIME-RESOLVED ARPES}

For the numerical evaluation of Eq. (8) we choose a VIS pulse, which resonantly excites the $1 s \mathrm{~A}$ exciton $(1.86 \mathrm{eV})$. The subsequent XUV probe pulse has an excitation energy of $20 \mathrm{eV}$ for the photoemission. The pump and probe pulse field intensity widths are 35 and $20 \mathrm{fs}$, respectively. The calculations are performed for an exemplary temperature of $77 \mathrm{~K}$ in the exemplary material $\mathrm{WSe}_{2}$ on a quartz substrate. For higher temperature we can expect a similar behavior in $\mathrm{WSe}_{2}$ except faster timescales due to a higher phonon occupation and more efficient exciton-phonon scattering [35]. Table I summarizes the used electronic and excitonic parameters.

\section{A. Excitonic signal at pulse overlap}

To address the coherent signals at pulse overlap first, Fig. 2 displays the result for monolayer $\mathrm{WSe}_{2}$ with a pulse delay of $25 \mathrm{fs}$ for the conduction and valence band region. Note that the $k_{\|}$axis has been shifted on top of the $K$ point. We discuss observed features (1)-(3) in Fig. 2 as follows:

(1) First, in Fig. 2(a) at $0 \mathrm{eV}$, the valence band electron dispersion can be recognized. This contribution results from the valence-band-vacuum transition $P_{\mathbf{k}_{\|}, \mathbf{k}}^{v f}$ in Eq. (17). In principle, at the band maximum a reduced trARPES signal reflects the optically excited hole distribution building up $1 s$ excitons. However, in the low-excitation regime considered here, this 

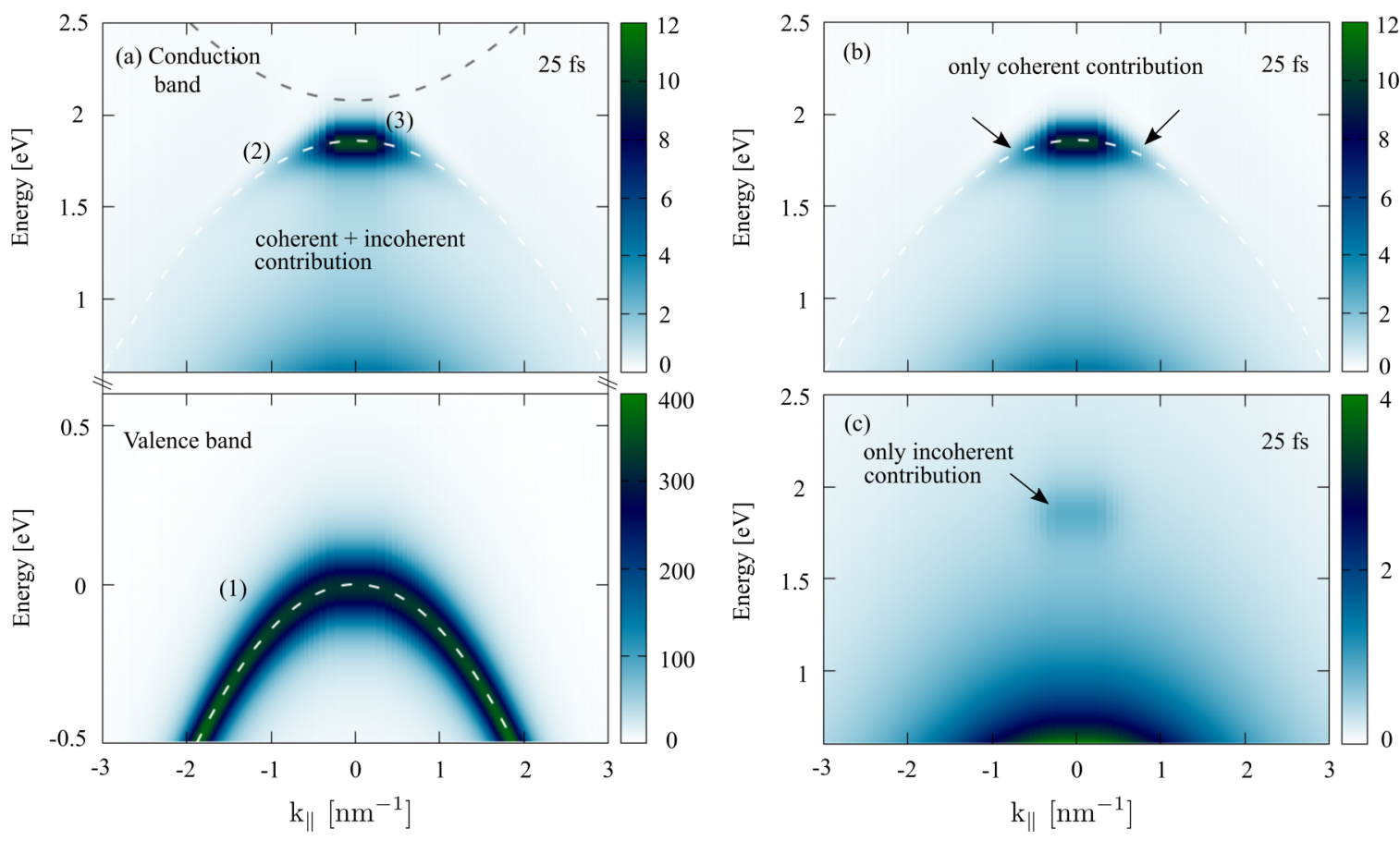

FIG. 2. The simulated photoemission signal of monolayer $\mathrm{WSe}_{2}$ at $77 \mathrm{~K}$ for a time delay of $25 \mathrm{fs}$. Here, (1), (2), and (3) refer to different aspects of the trARPES signal, which are discussed in Sec. (IV). (a) Conduction band region showing an excitonic signal stemming from coherent and incoherent $K K$ excitons at the exciton energy. The shadow of the valence band at the exciton energy reflects the presence of the coherent exciton. The gray dashed lines indicate the conduction band at the free-particle band gap, and the white dashed line sketches the valence band dispersion at the exciton energy. At $0 \mathrm{eV}$ the fully occupied valence band is visible. (b) Coherent contribution to the trARPES signal in the conduction band region. (c) Incoherent contribution to the trARPES signal in the conduction band region.

contribution is vanishingly small and cannot be seen in the plot.

(2) Second, in Figs. 2(a) and 2(b), we find a coherent excitonic signal at the exciton energy, which features a strong exciton contribution and a weak shadow of the valence band dispersion highlighted by the white dashed line at the exciton energy. To clarify the coherent contribution to the trARPES signal Fig. 2(b) displays the result for the conduction band region when setting the incoherent excitonic densities to zero, $N_{\mathbf{Q}}^{\xi_{v} \xi_{c}}=0$. Consequently, only the first two terms of Eq. (17) are nonvanishing. This coherent feature results from the coupling of the optically induced coherent excitonic transition $P_{0}^{\xi_{v} \xi_{v}}(\mathbf{Q}=0$ in the coherent limit) and the photoemission transition $P_{\mathbf{k}_{\|}, \mathbf{k}}^{v f \xi_{v}}$ between the valence band and vacuum states, [see Eq. (17), second term]. Since the optically excited excitonic transition exists only for vanishing center of mass momentum, this shadow of the valence band is visible only at the $K$ point. The valence band shadow is illustrated by arrows in Fig. 2(b). The decrease of the valence band shadow along the in-plane momentum is determined by the wave number decay of the exciton wave function $\left|\varphi_{\mathbf{q} \|}\right|^{2}$, which is obvious from the coupling element equation (17). Therefore, from Fig. 2, we conclude that in the ultrafast coherent limit trARPES is a technique to image the exciton wave function and measure the exciton Bohr radius in momentum space [33]. To obtain more analytical insights into the dominating excitonic signal, we can formally integrate Eqs. (16), (17), and (19) assuming exponentially shaped pulses of the form $\exp (-|t-\tau| / \sigma)$ with width $\sigma$. From the TMDC interband
Coulomb contribution we obtain a resonance of the signal [Eq. (8)] at $I_{\mathbf{k}_{\|}, \varepsilon_{\mathbf{k}}^{f}} \propto\left|\varphi_{\mathbf{k}_{\|}}\right|^{2} \rho_{\mathbf{k}_{\|}}^{v} \delta\left(\varepsilon_{\mathbf{k}}^{f}-\varepsilon_{\text {xuv }}-E_{0}^{1 s}-\varepsilon_{\mathbf{k}_{\|}}^{v}\right)$, where we assumed vanishing dephasing rates to use strict energy conservation. Clearly, the trARPES signal scales in $k_{\|}$with the exciton wave function $\left|\varphi_{\mathbf{k}_{\|}}\right|^{2}$.

(3) Third, in Figs. 2(a) and 2(c), incoherent $K K$ excitons, localized at the exciton energy, also generate an excitonic contribution in trARPES. The corresponding signal in Fig. 2(a) is, like the coherent signal (2), also downshifted by the binding energy with respect to the single-particle band gap (dashed line above $2 \mathrm{eV}$ ). Figure 2(c) shows the trARPES result for the incoherent limit with vanishing excitonic transition $P_{0}^{\xi_{v} \xi_{c}}=0$. The exciton population $N_{\mathbf{Q}}^{\xi_{v} \xi_{c}}$, which is the source of this signal, is determined by the exciton-phonon-induced scattering transfer of optically excited coherent to incoherent excitons [82]. The corresponding equation for $N_{\mathbf{Q}}^{\xi_{v} \xi_{c}}$ can be found in Ref. [35]. We consider the high-symmetry points $K, K^{\prime}, \Lambda$, and $\Lambda^{\prime}$ to compute the exciton formation and relaxation throughout the excitonic Brillouin zone. Therefore, the correlated two-particle quantity $\delta\left\langle P_{\mathbf{Q}}^{\dagger \xi_{c} \xi_{v}} P_{\mathbf{k}_{\|}-\mathbf{Q}, \mathbf{k}}^{v \xi_{v}}\right\rangle$ in Eq. (17) contains information about the exciton dynamics, namely, formation and relaxation. Obviously, the correlated excitonassisted photoemission quantity determines the signal by a convolution of the exciton wave function and distribution [see Eqs. (17) and (18)] when inserting the definitions for $\Omega_{\mu, \mathbf{k}, \mathbf{Q}}^{f c \xi_{c}}$ and $\tilde{\Omega}_{\mu, \lambda, \mathbf{k}, \mathbf{Q}}^{f c \xi_{v} \xi_{c}}$ right below. Together with the sum over the center of mass momentum along the exciton dispersion the trARPES signal does not display anymore the exact valence band shadow at the exciton energy. 


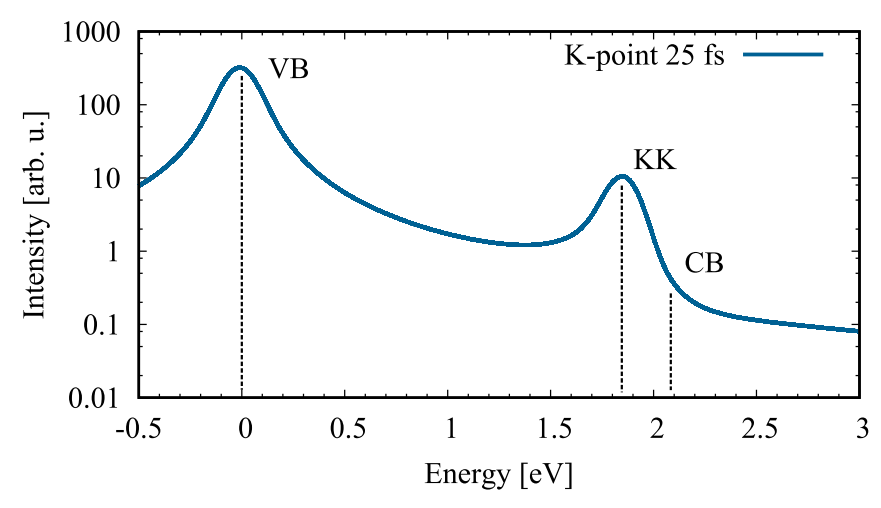

FIG. 3. Energy distribution curve in the low-excitation regime for $\mathrm{WSe}_{2}$ at the $K$ point 25 fs after optical excitation. Besides the valence band we find a peak at the exciton energy from the photoemission of conduction band electrons, forming here mainly coherent but also incoherent $K K$ excitons. Note the logarithmic scale.

We conclude that the excitonic signal (2) in Fig. 2 has two excitonic contributions, a coherent one and an incoherent one. But the main contribution for such short delay times stems from the coherent exciton since the incoherent excitons first have to be built up through phonon-induced dephasing from the excitonic transition [35].

Figure 3 shows the trARPES intensity for a fixed in-plane momentum $k_{\|}$, typically referred to as the energy distribution curve (EDC), at the $K$ point. As expected from Fig. 2, we observe two peaks, the first displaying the valence band and the second showing the exciton. The energetic position of the conduction band is also depicted as a dashed line for comparison. The shown result is valid for the low-excitation regime, reflected by the weak exciton signal compared to the valence band. Expanding the photoemission term of the valence band electron in Eq. (17) by inserting a unit operator $\sum_{\xi_{c}, \mathbf{k}_{\|}^{c}} c_{\mathbf{k}_{\|}^{\dagger} \xi_{c}}^{\xi_{\mathbf{k}_{\|}^{c}}^{\xi_{c}}}$ leads to

$$
\begin{aligned}
P_{\mathbf{k}_{\|}, \mathbf{k}}^{v f \xi_{v}}= & P_{\mathbf{k}_{\|}, \mathbf{k}}^{v f \xi_{v}}-\sum_{\mu, \xi_{c}} \varphi_{\mathbf{k}_{\|}}^{\xi_{v} \xi_{c}} P_{\mu, 0}^{\xi_{v} \xi_{c}} P_{\mathbf{k}_{\|}, \mathbf{k}}^{c f \xi_{c}} \\
& -\sum_{\mu, \xi_{c}, \mathbf{Q}} \varphi_{\mu, \mathbf{k}_{\|}+\beta_{\xi_{v}}^{\xi_{c}} \mathbf{Q}}^{\xi_{v} \xi_{c}} \delta\left\langle P_{\mu, \mathbf{Q}}^{\xi_{v} \xi_{c}} P_{\mathbf{k}_{\|}+\mathbf{Q}, \mathbf{k}}^{c f \xi_{c}}\right\rangle,
\end{aligned}
$$

resulting in excitonic corrections to the dominating electronic contribution of the valence band $P_{\mathbf{k}_{\|}, \mathbf{k}}^{v f \xi_{v}}$. The appearing correlated two-particle quantity, which is driven by the incoherent excitons, would lead to a weak excitonic satellite at the exciton binding energy above the valence band [32]. However, as already discussed, we focus on the low-excitation regime with $N_{\mathbf{Q}}^{\xi_{v} \xi_{c}} \ll 1$ and neglect these corrections.

\section{B. Exciton signals at large pulse delays}

So far we have investigated only short time delays and have focused on the optically excited $K$ point. Figures 4(a) and 4(b) display the result for a time delay much larger than the typical exciton-phonon scattering times, here taken as $400 \mathrm{fs}$. Because of the exciton-phonon scattering, side valleys, such as the $\Lambda$ valleys $\left(k_{\|} \approx 6 \mathrm{~nm}^{-1}\right)$ or $K^{\prime}$ valley, get populated. The reason is that the formation of momentum-indirect excitons, with a hole at the $K$ point and an electron at the $\Lambda$ or $K^{\prime}$ point, is energetically more favorable than the direct $K K$ excitons. The momentum-indirect incoherent $K \Lambda$ excitons can directly be observed at the $K \Lambda$ exciton energy below the conduction band at the $\Lambda$ point $\left(k_{\|} \approx 6 \mathrm{~nm}^{-1}\right)$ in time-resolved ARPES. Note that we show the trARPES signal as a function of the electronic wave number's absolute value. Therefore, all three $\Lambda$ valleys of the first Brillouin zone contribute to the same trARPES signal at $k_{\|}=\Lambda$. The $K K^{\prime}$ excitons have center of mass momentum $\mathbf{Q}=\mathbf{K}$. Therefore, in trARPES, where we investigate the signal as a function of the absolute value of the electronic momentum $k_{\|}$, the $K K^{\prime}$ exciton overlaps in
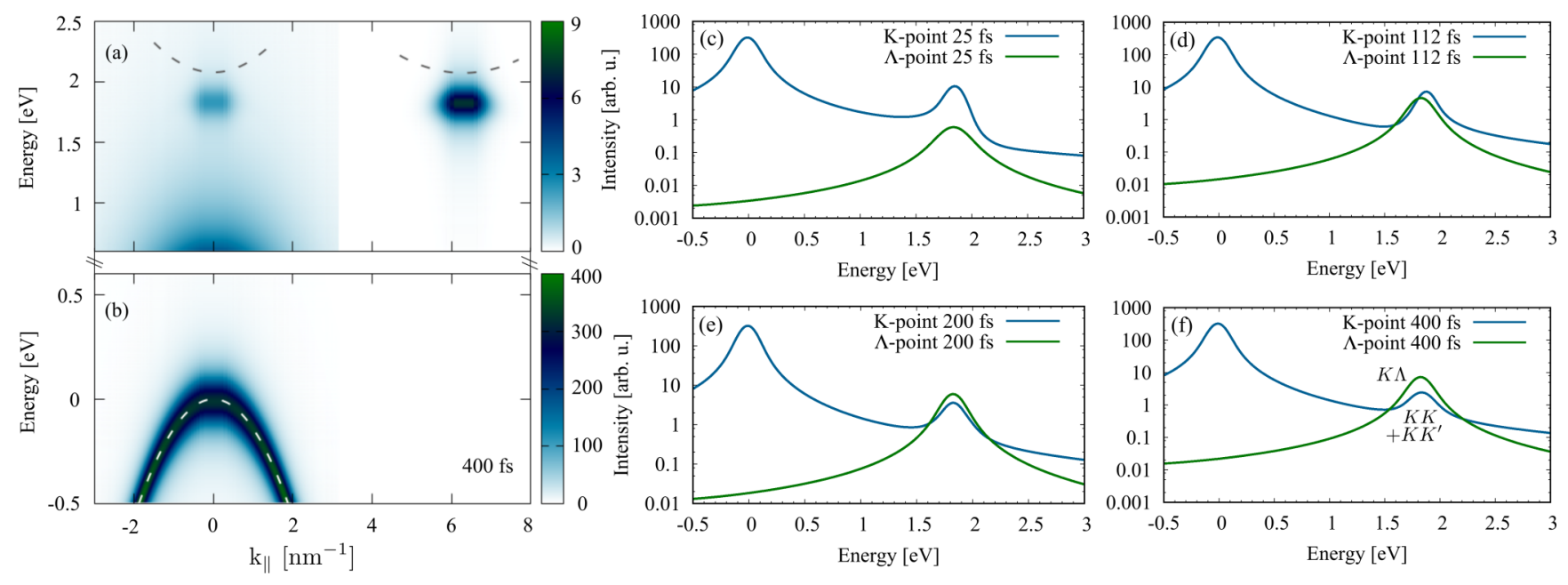

FIG. 4. The simulated photoemission signal of monolayer $\mathrm{WSe}_{2}$ at $77 \mathrm{~K}$ for a time delay of $400 \mathrm{fs}$ and EDCs for different time delays. (a) We observe the relaxation of electrons into the $\Lambda$ valley, forming momentum-indirect and energetically more favorable $K \Lambda$ excitons visible as an excitonic peak below the $\Lambda$ conduction band (dashed) at $k_{\|} \approx 6 \mathrm{~nm}^{-1}$. An excitonic signal remains at the $K$ point due to the formation of also energetically more favorable momentum-indirect $K K^{\prime}$ excitons. (b) The almost unperturbed valence band. (c)-(f) Energy distribution curves for time delays of 25,112, 200, and 400 fs showing the relaxation and thermalization of excitons into momentum-indirect excitonic states. 
momentum space with the $K K$ exciton, and its contribution to the trARPES signal lies at the $K$ point. In contrast to very short delay times, at large delays the signal is determined by the presence of incoherent excitons, and incoherent excitonphonon scattering can be investigated. Note that the exciton dynamics fulfill a detailed balance between coherent excitons $\left|P_{0}^{\xi_{0} \xi_{c}}\right|^{2}$, incoherent excitons $N_{\mathbf{Q}}^{\xi_{0} \xi_{c}}$, and the radiative decay.

The relaxation into $K \Lambda$ states can be seen more clearly by investigating the energy distribution curves in Fig. 4 for different consecutive pump-probe delay times around $1.8 \mathrm{eV}$. Within approximately the first 100 fs we observe the formation of incoherent excitons [see Figs. 4(c) and 4(d)] and then the subsequent thermalization [see Figs. 4(e) and 4(f)].

A different picture can be found in molybdenum-based TMDCs. Here, due to our calculations the $K K$ exciton state is the global minimum. Consequently, only intravalley excitonphonon scattering, mainly with acoustic phonons, takes place, leading to a thermalization of excitons [35] and a trARPES signal at the $K$ point.

\section{CONCLUSION}

In conclusion, we developed a theory of excitonic timeresolved ARPES in the low-excitation limit. We found that interband electron-hole Coulomb interaction strongly influences the photoemission spectrum, and therefore, we observed excitonic features in photoemission. We demonstrated that the photoemission through the optically injected excitonic transitions leads to unintuitive signals, namely, a shadow of the valence band at the excitonic energy. Additionally, we revealed that trARPES is able to probe the exciton dynamics and the exciton Bohr radius in TMDCs. We expect that our results are scalable to other two-dimensional structures such as van der Waals heterostructures, where more complex exciton dynamics takes place, namely, through energy or charge transfer of the excitons from one layer to another [83-85].

\section{ACKNOWLEDGMENTS}

We acknowledge financial support from the Deutsche Forschungsgemeinschaft (DFG) through SFB 951 (A.K., R.E., M.S., D.C.) Project No. 182087777. This project has also received funding from the European Unions Horizon 2020 research and innovation program under Grant Agreements No. 785219 (Graphene Flagship, E.M.) and No. 734690 (SONAR, A.K.). E.M. also acknowledges support from the Swedish Research Council (VR). R.E. acknowledges funding from the European Research Council (ERC) under the European Union's Horizon 2020 research and innovation program (Grant Agreement No. ERC-2015- CoG-682843). D.C. thanks the School of Nanophotonics (SFB 787 Project No. 43659573) for support. Finally, we thank F. Katsch, S. Kuhn, and M. Richter (TU Berlin) for valuable discussions.
[1] K. F. Mak, C. Lee, J. Hone, J. Shan, and T. F. Heinz, Phys. Rev. Lett. 105, 136805 (2010).

[2] A. Splendiani, L. Sun, Y. Zhang, T. Li, J. Kim, C.-Y. Chim, G. Galli, and F. Wang, Nano Lett. 10, 1271 (2010).

[3] A. Ramasubramaniam, Phys. Rev. B 86, 115409 (2012).

[4] K. He, N. Kumar, L. Zhao, Z. Wang, K. F. Mak, H. Zhao, and J. Shan, Phys. Rev. Lett. 113, 026803 (2014).

[5] A. Chernikov, T. C. Berkelbach, H. M. Hill, A. Rigosi, Y. Li, O. B. Aslan, D. R. Reichman, M. S. Hybertsen, and T. F. Heinz, Phys. Rev. Lett. 113, 076802 (2014).

[6] G. Wang, A. Chernikov, M. M. Glazov, T. F. Heinz, X. Marie, T. Amand, and B. Urbaszek, Rev. Mod. Phys. 90, 021001 (2018).

[7] T. Mueller and E. Malic, npj 2D Mater. Appl. 2, 29 (2018).

[8] A. Steinhoff, J.-H. Kim, F. Jahnke, M. Rösner, D.-S. Kim, C. Lee, G. H. Han, M. S. Jeong, T. O. Wehling, and C. Gies, Nano Lett. 15, 6841 (2015).

[9] A. Kormányos, G. Burkard, M. Gmitra, J. Fabian, V. Zólyomi, N. D. Drummond, and V. Fal'ko, 2D Mater. 2, 022001 (2015).

[10] F. A. Rasmussen and K. S. Thygesen, J. Phys. Chem. C 119, 13169 (2015).

[11] A. Molina-Sánchez, M. Palummo, A. Marini, and L. Wirtz, Phys. Rev. B 93, 155435 (2016).

[12] F. Wu, F. Qu, and A. H. MacDonald, Phys. Rev. B 91, 075310 (2015).

[13] D. Y. Qiu, T. Cao, and S. G. Louie, Phys. Rev. Lett. 115, 176801 (2015).

[14] M. Selig, G. Berghaeuser, A. Raja, P. Nagler, C. Schüller, T. F. Heinz, T. Korn, A. Chernikov, E. Malic, and A. Knorr, Nat. Commun. 7, 13279 (2016).
[15] E. Malic, M. Selig, M. Feierabend, S. Brem, D. Christiansen, F. Wendler, A. Knorr, and G. Berghäuser, Phys. Rev. Mater. 2, 014002 (2018).

[16] J. P. Echeverry, B. Urbaszek, T. Amand, X. Marie, and I. C. Gerber, Phys. Rev. B 93, 121107(R) (2016).

[17] R. Schmidt, G. Berghäuser, R. Schneider, M. Selig, P. Tonndorf, E. Malić, A. Knorr, S. M. de Vasconcellos, and R. Bratschitsch, Nano Lett. 163, 2945 (2016).

[18] P. Steinleitner, P. Merkl, A. Graf, P. Nagler, K. Watanabe, T. Taniguchi, J. Zipfel, C. Schüller, T. Korn, A. Chernikov, S. Brem, M. Selig, G. Berghäuser, E. Malic, and R. Huber, Nano Lett. 18, 1402 (2018).

[19] M. Selig, F. Katsch, R. Schmidt, S. M. de Vasconcellos, R. Bratschitsch, E. Malic, and A. Knorr, Phys. Rev. Research 1, 022007(R) (2019).

[20] X.-X. Zhang, Y. You, S. Y. F. Zhao, and T. F. Heinz, Phys. Rev. Lett. 115, 257403 (2015).

[21] J. Lindlau, C. Robert, V. Funk, J. Förste, M. Förg, L. Colombier, A. Neumann, E. Courtade, S. Shree, T. Taniguchi, K. Watanabe, M. M. Glazov, X. Marie, B. Urbaszek, and A. Högele, arXiv:1710.00988.

[22] J. Lindlau, M. Selig, A. Neumann, L. Colombier, J. Förste, V. Funk, M. Förg, J. Kim, G. Berghäuser, T. Taniguchi, K. Watanabe, F. Wang, E. Malic, and A. Högele, Nat. Commun. 9, 2586 (2018).

[23] R. Bertoni, C. W. Nicholson, L. Waldecker, H. Hübener, C. Monney, U. De Giovannini, M. Puppin, M. Hoesch, E. Springate, R. T. Chapman, C. Cacho, M. Wolf, A. Rubio, and R. Ernstorfer, Phys. Rev. Lett. 117, 277201 (2016). 
[24] R. Wallauer, J. Reimann, N. Armbrust, J. Güdde, and U. Höfer, Appl. Phys. Lett. 109, 162102 (2016).

[25] M. Puppin, Y. Deng, C. W. Nicholson, J. Feldl, N. B. M. Schröter, H. Vita, P. S. Kirchmann, C. Monney, L. Rettig, M. Wolf, and R. Ernstorfer, Rev. Sci. Instrum. 90, 023104 (2019).

[26] A. Damascelli, Z. Hussain, and Z. Shen, Rev. Mod. Phys. 75, 473 (2003).

[27] L. Töben, L. Gundlach, R. Ernstorfer, R. Eichberger, T. Hannappel, F. Willig, A. Zeiser, J. Förstner, A. Knorr, P. H. Hahn, and W. G. Schmidt, Phys. Rev. Lett. 94, 067601 (2005).

[28] J. Güdde, M. Rohleder, T. Meier, S. W. Koch, and U. Höfer, Science 318, 1287 (2007).

[29] T. Suzuki and R. Shimano, Phys. Rev. Lett. 103, 057401 (2009).

[30] H. Wang, C. Zhang, and F. Rana, Nano Lett. 15, 339 (2015).

[31] E. Perfetto, D. Sangalli, A. Marini, and G. Stefanucci, Phys. Rev. B 94, 245303 (2016).

[32] A. Steinhoff, M. Florian, M. Rösner, G. Schönhoff, T. O. Wehling, and F. Jahnke, Nat. Commun. 8, 1166 (2017).

[33] A. Rustagi and A. F. Kemper, Phys. Rev. B 97, 235310 (2018).

[34] A. Rustagi and A. F. Kemper, Phys. Rev. B 99, 125303 (2019).

[35] M. Selig, G. Berghäuser, M. Richter, R. Bratschitsch, A. Knorr, and E. Malic, 2D Mater. 5, 035017 (2018).

[36] D. Christiansen, M. Selig, G. Berghäuser, R. Schmidt, I. Niehues, R. Schneider, A. Arora, S. M. de Vasconcellos, R. Bratschitsch, E. Malic, and A. Knorr, Phys. Rev. Lett. 119, 187402 (2017).

[37] A. Raja, M. Selig, G. Berghäuser, J. Yu, H. M. Hill, A. F. Rigosi, L. E. Brus, A. Knorr, T. F. Heinz, E. Malic, A. Chernikov, Nano Lett. 18, 6135 (2018).

[38] C. Timm and K. H. Bennemann, J. Phys.: Condens. Matter 16, 661 (2004).

[39] M. Sakaue, J. Phys.: Condens. Matter 17, 245 (2005).

[40] H. Ueba and B. Gumhalter, Prog. Surf. Sci. 82, 193 (2007).

[41] A. Zeiser, N. Bücking, J. Förstner, and A. Knorr, Phys. Rev. B 71, 245309 (2005).

[42] M. Wolf, A. Hotzel, E. Knoesel, and D. Velic, Phys. Rev. B 59, 5926 (1999).

[43] M. Weinelt, J. Phys.: Condens. Matter 14, R1099 (2002).

[44] G. Dresselhaus, J. Phys. Chem. Solids. 1, 14 (1956).

[45] R. J. Elliott, Phys. Rev. 108, 1384 (1957).

[46] H. Haug and S. W. Koch, Quantum Theory of the Optical and Electronic Properties of Semiconductors, 5th ed. (World Scientific, Singapore, 2004).

[47] H. Haken, Quantum Field Theory of Solids: An Introduction (North-Holland, Amsterdam, New York, Oxford, 1976).

[48] M. L. Trolle, T. G. Pedersen, and V. Véniard, Sci. Rep. 7, 39844 (2017)

[49] N. S. Rytova, Moscow Univ. Phys. Bull. 22, 18 (1967).

[50] L. V. Keldysh, JETP Lett. 29, 658 (1978).

[51] T. C. Berkelbach, M. S. Hybertsen, and D. R. Reichman, Phys. Rev. B 88, 045318 (2013).

[52] A. Steinhoff, T. O. Wehling, and M. Rösner, Phys. Rev. B 98, 045304 (2018).

[53] B. Scharf, D. V. Tuan, I. Žutić, and H. Dery, J. Phys.: Condens. Matter 31, 203001 (2019).

[54] X. Cui, C. Wang, A. Argondizzo, S. Garrett-Roe, B. Gumhalter, and H. Petek, Nat. Phys. 10, 505 (2014).

[55] V. M. Silkin, P. Lazić, N. Došlić, H. Petek, and B. Gumhalter, Phys. Rev. B 92, 155405 (2015).
[56] See Supplemental Material at http://link.aps.org/supplemental/ 10.1103/PhysRevB.100.205401 for detailed information about the electronic wave functions, the transition dipole element, and the Coulomb matrix element.

[57] Z. Jin, X. Li, J. T. Mullen, and K. W. Kim, Phys. Rev. B 90, 045422 (2014).

[58] X. Li, J. T. Mullen, Z. Jin, K. M. Borysenko, M. Buongiorno Nardelli, and K. W. Kim, Phys. Rev. B 87, 115418 (2013).

[59] K. Kaasbjerg, K. S. Thygesen, and K. W. Jacobsen, Phys. Rev. B 85, 115317 (2012).

[60] K. Kaasbjerg, K. S. Thygesen, and A.-P. Jauho, Phys. Rev. B 87, 235312 (2013).

[61] Y. Guo and J. Robertson, Appl. Phys. Lett. 108, 233104 (2016).

[62] K. Keyshar, M. Berg, X. Zhang, R. Vajtai, G. Gupta, C. K. Chan, T. E. Beechem, P. M. Ajayan, A. D. Mohite, and T. Ohta, ACS Nano 11, 8223 (2017).

[63] See Supplemental Material at http://link.aps.org/supplemental/ 10.1103/PhysRevB.100.205401 for detailed information about the optical matrix element between TMDC and vacuum states.

[64] J. K. Freericks, H. R. Krishnamurthy, and T. Pruschke, Phys. Rev. Lett. 102, 136401 (2009).

[65] S. Ramakrishna, F. Willig, and A. Knorr, Surf. Sci. 558, 159 (2004).

[66] M. Eckstein and M. Kollar, Phys. Rev. B 78, 245113 (2008).

[67] J. Braun, R. Rausch, M. Potthoff, J. Minár, and H. Ebert, Phys. Rev. B 91, 035119 (2015).

[68] H. Petek and S. Ogawa, Prog. Surf. Sci. 56, 239 (1997).

[69] B. Gumhalter, in Encyclopedia of Interfacial Chemistry: Surface Science and Electrochemistry, edited by K. Wandelt (Elsevier, Amsterdam, 2018), pp. 668-685.

[70] M. Kira, F. Jahnke, W. Hoyer, and S. W. Koch, Prog. Quantum Electron. 23, 189(1999).

[71] T. Usui, Prog. Theor. Phys. 23, 787 (1960).

[72] A. L. Ivanov and H. Haug, Phys. Rev. B 48, 1490 (1993).

[73] A. J. Fischer, D. S. Kim, J. Hays, W. Shan, J. J. Song, D. B. Eason, J. Ren, J. F. Schetzina, H. Luo, J. K. Furdyna, Z. Q. Zhu, T. Yao, J. F. Klem, and W. Schäfer, Phys. Rev. Lett. 73, 2368 (1994).

[74] F. Katsch, M. Selig, A. Carmele, and A. Knorr, Phys. Status Solidi B 255, 1800185 (2018).

[75] V. Axt and A. Stahl, Z. Phys. B 93, 195 (1994).

[76] M. Lindberg, Y. Z. Hu, R. Binder, and S. W. Koch, Phys. Rev. B 50, 18060 (1994).

[77] J. Fricke, Ann. Phys. (NY) 252, 479 (1996).

[78] M. Kira and S. Koch, Prog. Quantum Electron. 30, 155 (2006).

[79] V. M. Axt and T. Kuhn, Rep. Prog. Phys. 67, 433 (2004).

[80] S. Brem, M. Selig, G. Berghaeuser, and E. Malic, Sci. Rep. 8, 8238 (2018).

[81] S. Brem, J. Zipfel, M. Selig, A. Raja, L. Waldecker, J. Ziegler, T. Taniguchi, K. Watanabe, A. Chernikov, and E. Malic, Nanoscale 11, 12381 (2019).

[82] A. Thränhardt, S. Kuckenburg, A. Knorr, T. Meier, and S. W. Koch, Phys. Rev. B 62, 2706 (2000).

[83] M. Selig, E. Malic, K. J. Ahn, N. Koch, and A. Knorr, Phys. Rev. B 99, 035420 (2019).

[84] S. Ovesen, S. Brem, C. Linderälv, M. Kuisma, T. Korn, P. Erhart, M. Selig, and E. Malic, Commun. Phys. 2, 23 (2019).

[85] S. Aeschlimann, A. Rossi, M. Chávez-Cervantes, R. Krause, B. Arnoldi, B. Stadtmüller, M. Aeschlimann, S. Forti, F. Fabbri, C. Coletti, and I. Gierz, arXiv:1904.01379. 